\title{
Neglected interstitial space in malaria recurrence and treatment
}

\author{
Qiang Zhang ${ }^{1,2}$, Zhuo Ao ${ }^{1,2}(\bowtie)$, Nan Hü ${ }^{1,3}$, Yuting Zhu' ${ }^{1}$, Fulong Liao ${ }^{1,4}$, and Dong $\operatorname{Han}^{1,2}(\bowtie)$ \\ ${ }^{1}$ CAS Center for Excellence in Nanoscience, National Center for Nanoscience and Technology, Beijing 100190, China \\ ${ }^{2}$ University of Chinese Academy of Sciences, Beijing 100049, China \\ ${ }^{3}$ Department of Traditional Chinese Medicine, Chengde Medical University, Chengde 066000, China \\ ${ }^{4}$ Artemisinin Research Center and the Institute of Chinese Materia Medica, China Academy of Chinese Medical Sciences, Beijing 100049, \\ China
}

(C) Tsinghua University Press and Springer-Verlag GmbH Germany, part of Springer Nature 2020

Received: 14 May 2020 / Revised: 15 June 2020 / Accepted: 19 June 2020

\begin{abstract}
The interstitial space, a widespread fluid-filled compartment throughout the body, is related to many pathophysiological alterations and diseases, attracting increasing attention. The vital role of interstitial space in malaria infection and treatment has been neglected current research efforts. We confirmed the reinfection capacity of parasites sequestrated in interstitial space, which replenish the mechanism of recurrence. Malaria parasite-infected mice were treated with artemisinin-loaded liposomes through the interstitial space and exhibited a better therapeutic response. Notably, compared with oral administration, interstitial administration showed an unexpectedly high activation and recruitment of immune cells, and resulted in better clearance of sequestered parasites from organs, and enhanced pathological recovery. The interstitial route of administration prolongs the blood circulation time of artemisinin and increases its plasma concentration, and may compensate for the inefficiency of oral administration and the nanotoxicity of intravenous administration, providing a potential strategy for infectious disease therapy.
\end{abstract}

\section{KEYWORDS}

interstitial space, malaria, infection, recurrence, treatment

\section{Introduction}

Complex living organisms have typical soft matter with structure-function units in hierarchical encapsulation of the interstitium. Among these, interstitial architectures are formed from large porous network of fibers (such as extracellular matrix, loose connective tissue matrix) into gradually dense fibrous network. The matrix gel is extruded between single fiber lamellae, and the multilayer assembly eventually forms the generalized fascia, and compresses into a tendon or surrounds the interstitial space, which can interpenetrate all organs and muscles. Blood vessels, lymphatic vessels, and nerve bundles pass through the interstitial space. The most real image of life activities is the interstitial structure that runs through the whole body, with an internal green pathway (interstitial stream) - the "hierarchical complex fluid-interstitial stream" life model-which makes the whole body become an organic whole of flattened connections [1].

The interstitial fluid can work as a shock absorber to protect organs and tissues $[2,3]$. The interstitial structure and composition vary in different organs, but are similar to some extent, consisting of flowing interstitial fluid and a complex network of collagen bundles [4]. In addition to nutritional and mechanical support functions, interstitial space can also serve as a passage for the transportation of cancer cells, immune cells and microorganisms, and may be involved in cancer metastasis, fibrosis, and edema $[4,5]$. In our previous studies, we used intervaginal space injection of different tracers to investigate the transportation behaviors of interstitial space, which confirm that certain interstitial space can work as a long-range and high efficiency connection route for mass delivery $[6,7]$. Due to a typical delayering connection, interstitial space can quickly and directly transport substances from intervaginal injection sites to organs and tissues, and can even reach the central nervous system bypassing the blood-brain barrier, which indicates a superior pathway for drug delivery to treat cerebral diseases [1]. Recent studies have demonstrated that the subcutaneous interstitium can act as a reservoir of fibroblasts and scar-forming provisional matrix to quickly seal large open wounds, providing new insights on diabetic and ulcerative wounds $[8,9]$. Besides, the cerebral interstitial space, surrounding arterioles and venules, play important roles in clearance of interstitial fluid and waste from the brain, and is known to be associated with aging, Alzheimer's disease, diabetes, and malaria [10-15].

Upon infection by Plasmodium, erythrocytes undergo extensive alterations in structural properties, resulting in increased membrane stiffness and viscosity [16]. Hence, the infected red blood cells (iRBCs) easily adhere onto vascular endothelium, activating leukocytes and endothelial cells. The sequestration of iRBCs leads to vascular obstruction and excessive secretion of cytokines, increasing vascular permeability [17]. Simultaneously, parasite-specific $\mathrm{CD}^{+} \mathrm{T}$ cells are recruited to the perivascular space, which damages the integrity of blood vessels [18]. The disrupted vascular integrity and increased vascular permeability induce vascular hemorrhage, accompanied by an enlargement of the perivascular space [19]. Many studies have observed that parasitized erythrocytes can be located within the interstitial space [18-21], which may cause a decrease in 
the clearance rate of iRBCs by the spleen and increases the probability of recurrence after recovery. It is crucial to eliminate parasites sequestrated in perivascular space; however, the relationship between the interstitial space and malaria treatment has been unclear.

Here, we explored the malaria infection process by interstitial injection of infected erythrocytes, compared with intravenous infection and intraperitoneal infection, evaluating the reinfection capacity of parasites sequestrated in interstitial space. Importantly, we treated infected mice with artemisinin-loaded liposomes (ALL) via interstitial space injection to investigate the potential of the interstitial space as a pathway in malaria therapy.

\section{Experimental}

\subsection{Animals and malaria model}

All animal experiments were approved by the Institutional Animal Care and Use Committee. BALB/c mice (male, $20 \pm 2 \mathrm{~g}$ ) were purchased from Beijing Vital River Laboratory Animal Technology Co., Ltd (Beijing, China). Different malaria infection mice models were established by intravenous injection, intraperitoneal injection, and intervaginal space injection (ISI) with iRBCs. Experimental mice were inoculated with $1 \times 10^{7}$ Plasmodium yoelii-infected erythrocytes in $0.1 \mathrm{~mL}$ saline solution. Infected erythrocytes stored at $-80{ }^{\circ} \mathrm{C}$ were first activated by inoculating donor mice. Thereafter, freshly infected blood was collected in anticoagulant tubes from the ocular venous sinus of donor mice and used to establish the malaria models.

\subsection{Preparation of ALL}

Thin film hydration method was used to prepare liposomes. Briefly, a mixture of lecithin, cholesterol, and artemisinin in a 25:5:7 mass ratio was dissolved in chloroform/methanol $(2: 1, \mathrm{v} / \mathrm{v})$ solution. Under reduced pressure and $40{ }^{\circ} \mathrm{C}$, the solution was evaporated to form a phospholipid film using a rotary evaporator (DLAB, China), which was subsequently dried under nitrogen. Thereafter, normal saline was added to hydrate the phospholipid film for $30 \mathrm{~min}$ at $60{ }^{\circ} \mathrm{C}$, followed by sonication for $15 \mathrm{~min}$ in a bath sonicator at $42 \mathrm{kHz}$ and $100 \mathrm{~W}$. The resulting liposomes were subsequently extruded through a $100 \mathrm{~nm}$ polycarbonate porous membrane using an extruder (Avestin, Canada).

\subsection{Characterization of ALL}

The size, polydispersity index, and zeta-potential of ALL were measured by dynamic light scattering (Malvern, UK). Measurements were performed in triplicate at room temperature. The morphology of liposomes was characterized by a transmission electron microscope (TEM) (Hitachi, Japan). A drop of diluted ALL was placed onto a carbon-coated grid and air-dried. Thereafter, a drop of $1 \%$ uranyl acetate stain was deposited onto the grid. The grid was subsequently dried and visualized under a TEM.

\subsection{Drug treatment}

After three days of infection with P. yoelii, mice with $30 \%$ parasitemia were randomly divided into three groups of six mice each. One group was treated intragastrically with artemisinin at a dosage of $100 \mathrm{mg} / \mathrm{kg}$, once a day, for six consecutive days. The other two groups were injected with an equivalent amount of ALL in $0.1 \mathrm{~mL}$ saline solution using intervaginal space injection at the upper limb and lower limb.

\subsection{Blood smear}

Once mice were infected with parasites, peripheral blood smears were made daily to monitor the growth status of the parasite. The tail was cut off a little and squeezed to form a drop of blood on a glass slide, and a thin film of blood was made by spreading the blood. Smears were air-dried and fixed with methanol. Subsequently, blood smears were stained in 10\% Giemsa solution (Solarbio, China) for $15 \mathrm{~min}$, rinsed with distilled water, and dried at room temperature. The blood smears were visualized under a light microscope with $100 \times$ oil immersion lens (Nikon, Japan). Parasitemia was calculated by counting the number of infected red blood cells per 1,000 cells.

\subsection{Hematological measurements}

Fresh blood samples were drawn from the ocular venous sinus and placed in ethylene diamine tetraacetic acid (EDTA) tubes (BD, USA). A total of $100 \mathrm{~L}$ of blood sample without coagulant was loaded onto an automatic cell counter (Tecom Science, China). Hematological parameters, such as red blood cells (RBCs) count, hematocrit, hemoglobin (HGB) concentration, lymphocyte (LYM) count, and white blood cell (WBC) count, were also obtained from the machine.

\subsection{Histopathological analysis}

Liver and spleen from mice of both infection and therapy models were harvested and fixed in $4 \%$ paraformaldehyde (Solarbio, China) for histopathological analysis. The samples were dehydrated using a graded series of alcohol, followed by embedding in paraffin wax. Thereafter, the samples were sectioned with a microtome (Leica, Germany), stained in hematoxylin and eosin solution (Solarbio, China), and visualized using light microscopy.

\subsection{Plasma concentration by LC-MS}

Normal healthy mice were divided into two groups for artemisinin treatment at a dosage of $100 \mathrm{mg} / \mathrm{kg}$. A group was treated with artemisinin by oral administration, and the other was treated with ALL by interstitial administration. Blood samples were drawn from the ocular venous sinus before dosing and at $0.5,1,1.5,2,4,6,8,16,24$ h post-dosing. All anticoagulative blood samples were centrifuged at $5,000 \mathrm{~g}$ for $15 \mathrm{~min}$ at $4{ }^{\circ} \mathrm{C}$ to obtain plasma. A mixture of $100 \mu \mathrm{L}$ of plasma and $400 \mu \mathrm{L}$ acetonitrile was centrifuged at $15,000 \mathrm{~g}$ at $4{ }^{\circ} \mathrm{C}$ for $15 \mathrm{~min}$. The supernatant was obtained and filtered through an organic filter membrane. Artemisinin standard was diluted with acetonitrile to give working solutions of 20,50, 100, 200, 500, 1,000, 2,000, $5,000 \mathrm{ng} / \mathrm{mL}$. Calibration standards were prepared by mixing $50 \mu \mathrm{L}$ of working solution and $100 \mu \mathrm{L}$ of drug-free plasma and $350 \mu \mathrm{L}$ acetonitrile. This mixture was treated as described above and $2 \mu \mathrm{L}$ of each sample was injected into an liquid chromatography-mass spectrometer (LC-MS) system for analysis.

The LC-MS analysis was performed using a LCMS-8050 triple quadrupole mass spectrometer (Shimadzu, Japan). Chromatographic separation was achieved on a C18 column (2.1 $\mathrm{mm} \times 150 \mathrm{~mm}, 3 \mu \mathrm{m}$, Shimadzu, Japan). The mobile phase consisted of acetonitrile $/ 0.1 \%$ formic acid $(85: 15, \mathrm{v} / \mathrm{v})$, delivered at a flow rate of $0.3 \mathrm{~mL} / \mathrm{min}$. Quantification was achieved using reaction monitoring for the transitions $\mathrm{m} / z 283$ to 265 .

\section{Results}

\subsection{Infection process by injection of interstitial space}

To verify the infectivity of parasites sequestrated in interstitial space, we injected $P$. yoelii-infected erythrocytes into interstitial space by ISI. Two injection sites of interstitial space were chosen 
in the carpal tunnel and the tarsal tunnel, according to our previous studies $[5,6]$.

ISI in the upper limb (i.s.u.) can reach axillary artery along the continuous channel (Fig. 1(a)). Similarly, ISI in the lower limb (i.s.l.) converge in the groin by following the perivascular space of the femoral artery or the saphenous vein (Fig. 1(b)). After infection by ISI, the site was resected and fixed for histological sectioning. These sections were stained with hematoxylin and eosin, and confirmed the presence of parasites in the reticular structure of interstitial space (Fig. 1(c)). The network is defined by collagen bundles, with blank spaces for liquid transportation. Parasites can also be witnessed outside the blood vessel, proving the successful injection into perivascular space (Fig. 1(d)). Besides, we also observed that malaria parasites were attempting to traverse the endothelial cells into the vascular lumen (Fig. 1(e)). After injection into intervaginal sites, parasites inside erythrocytes can progress through their erythrocytic stage. Once matured, merozoites released from red blood cells can complete their intact life cycle by invading healthy red blood cells leaked in interstitial space or attempt to enter into blood vessels and return back to blood circulation. Merozoites can quickly move to capillaries along interstitial space based on the delayering connection, which makes it easier for parasites to invade into thin vascular wall (Fig. 1(f)). Once in the blood vessel, parasites can start their new life cycle in blood circulation.

Interstitial system wreathes and interpenetrates all organs, muscles, blood vessels, and neuropil, playing vital roles in architectural function, morphogenesis and signal transmission. The interstitial membrane (generalized fascia) works as a strap tube that surrounds vessels and nerves (Fig. 1(f)), separating the neurovascular bundle transmission systems from surrounding tissues. These inclusions inside interstitial space can work independently, but affect each other. Nerves can secrete hormones and neurotransmitters that affect the behavior of blood vessels and interstitial stream, and vice versa, the mass transportation of vascular system and interstitial stream give feedback to the nervous system. Proteins and free cells can leak out from vessels into interstitial space to regulate osmotic pressure and pathological conditions of the interstitial system. Meanwhile, the alteration of conformation and mechanical properties of interstitial protein fiber can influence the condition of blood flow. Hence, it is nonnegligible to take interstitial system into consideration when diseases take place. Interstitial space can act as a transportation pathway as blood vessels, but they are not only different in the substances transported but also in the way of transport. As an interconnected whole, the human body relies on connective tissue to separate and connect functional structures. The blood is the main line of nutrition and substances transportation. In order to connect all living cells, the aorta needs to be divided into arterioles. Most of blood vessels are bifurcated, as many bifurcations are needed to fill the whole space. The fractal dimension of vascular network of multiple organs varies from 1.8 to 2.8 due to different organ properties and calculation methods [22]. In contrast, the generalized fascia system is wrapped around the tissue, which itself is a folded two-dimensional membrane, and it usually expands from a point to a multi-level wrapping structure. However, there are still no reports about the multi-level connection structure of fascial anatomy, and its fractal dimension has not been calculated. However, its connection distance should be less than the connection distance through the vascular network because the fractal dimension of its expansion characteristics should be less than 2 . In addition, the number of bifurcations in each stage is far greater than 2 , and the total number of bifurcations is far less than the number of vessels, similar to the delayering tissue structure, which further reduces the level of connectivity between different structures. At the same time, the tissue expands outwards and increases the surface area under the shackles of generalized fascia. Therefore, when injected at the interstitial convergence position, the expansion of the membrane area and negative pressure will cause the injected substances to flow to the enlarged tissue, such that it spontaneously gather around cell clusters and more easily invade the capillary network.

\subsection{Comparison of infection rate by different injection methods}

In order to verify the validity of interstitial injection, mice were also intravenously (i.v.) and intraperitoneally (i.p.) injected with $P$. yoelii-infected erythrocytes, and the infection rate was compared with i.s.u. and i.s.l. for seven consecutive days. Blood smears were prepared every day to monitor the infection rate (Fig. 2(a)). In the first two days, plasmodia could barely be seen in blood smears of all four groups. Although larger amounts of plasmodia were directly injected into the blood by i.v., most of the organisms were eliminated by the macrophages or the spleen, while the rest hid themselves, resulting in the disappearance of iRBCs from the blood on the first day. On
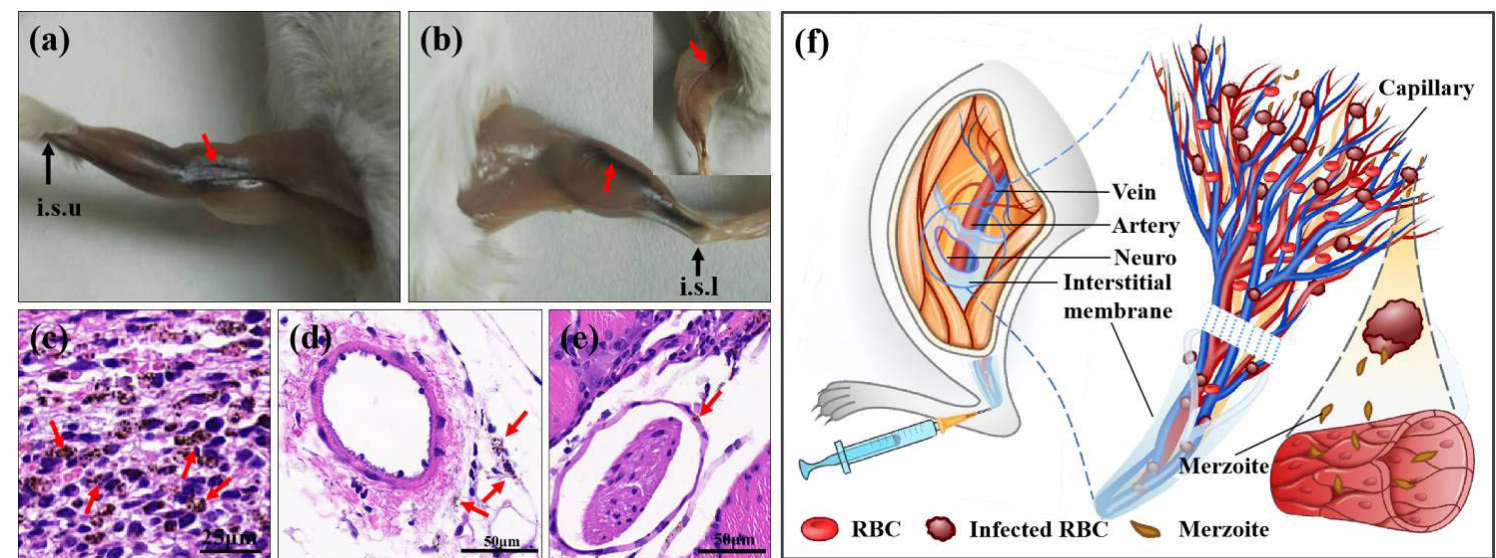

Figure 1 Infection by interstitial space injection. (a) Model was established by interstitial space injection in the upper limb (i.s.u.). Fluid can transport along the space from injection site (red arrow) to axillary artery. (b) Model was established by interstitial space injection in lower limb (i.s.l.). The black infected blood can transport along femoral artery (red arrow) or saphenous vein (as showed in inset image) from injection site (black arrow) to groin (red arrow). (c) Malaria parasites (brown, red arrow) were distributed in the reticular structure. (d) Malaria parasites (brown, red arrow) appeared outside a blood vessel. (e) Parasites (brown, red arrow), trying to invade a blood vessel, and enter blood circulation. (f) Schematic representation of interstitial infection. 

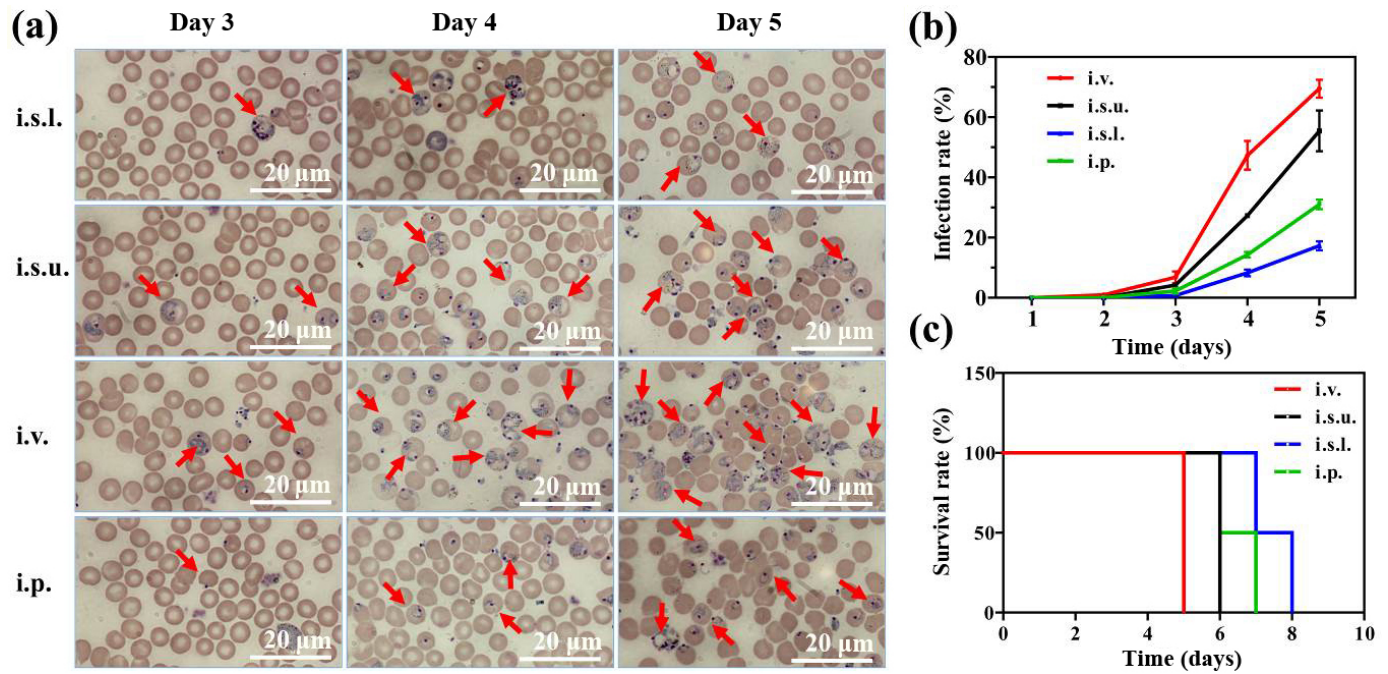

Figure 2 Comparison of different infection methods. (a) Blood smear images of 3-5 days after infection for different injection methods. Infected red blood cells were marked with red arrow. (b) Infection rate on five consecutive days. (c) Survival rate of mice infected using different injection methods.

the third day after injection, iRBCs could be observed in the blood. The i.v. group exhibited the highest amounts of iRBCs, while less than five iRBCs could be seen in one image of the other three groups. Four days post-infection, significant differences were seen among the four groups. The i.s.u. group showed unexpectedly large amounts of iRBCs, exceeding that observed in the i.p. group, while the i.s.l. group had the least number of iRBCs.

As shown in Fig. 2(b), the i.v. group exhibited the highest infection rate, followed by the i.s.u. group. Malaria parasites injected by i.s.u. could easily arrive and invade into axillary artery, resulting in high similarity with i.v.. However, injection in the lower limb was a longer way to transport parasites to the liver and easier to be sequestrated halfway by the spleen. Hence, the i.s.l. group showed the lowest infection rate during the infection period. After five days of infection, the parasitemia of the i.v. group reached up to $70 \%$, while that of the i.s.l. group was less than $20 \%$. Due to high parasitemia, mice of the i.v. group died before the sixth day (Fig. 2(c)). As the parasitemia became more and more serious, all mice died. The mice of i.s.l. group survived up to day 8 with an exponential growth of infection rate. Parasites injected by i.v. directly entered the blood vessels, while parasites injected by i.p. first invaded the hepatocytes.

\subsection{Hematological alterations of interstitial infection}

Hematological parameters such as RBC count, HGB concentration, LYM count, and WBC count are important indicators for disease diagnosis and treatment and can sensitively reflect pathological changes and disease progression in the body. We monitored these parameters daily to evaluate the degree of infection (Fig. 3). Consistent with results of the blood smear, there was no significant decrease in RBC count in the first two days. A noticeable change was seen from day 3 . The i.s.u. group exhibited a faster decline in RBC count and HGB concentration than the i.s.l. group (Figs. 3(a) and 3(b)), indicating the occurrence of massive growth of malaria parasites. As the condition deteriorated, the RBC count decreased to $2 \times 10^{12} / \mathrm{L}$, resulting in severe anemia and even death.

In contrast, WBC and LYM counts increased with time (Figs. 3(c) and 3(d)). During the first three days, there were no obvious alterations due to the absence of parasites in blood circulation due to the immune evasion by the parasites. Moreover, we observed that the WBC and LYM counts increased faster in the i.s.u. group compared to the i.s.u. group. The WBC count of the i.s.u. group increased five times than the normal value (a)

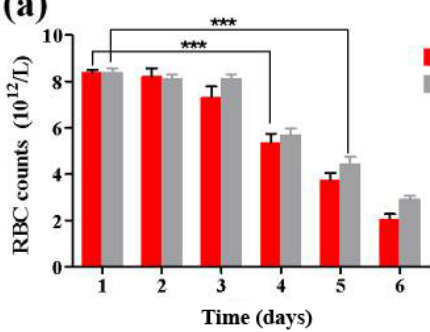

(c)

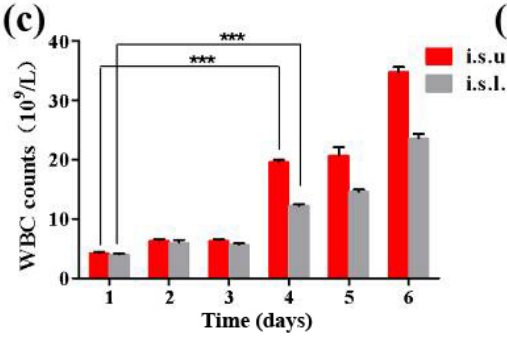

(b)

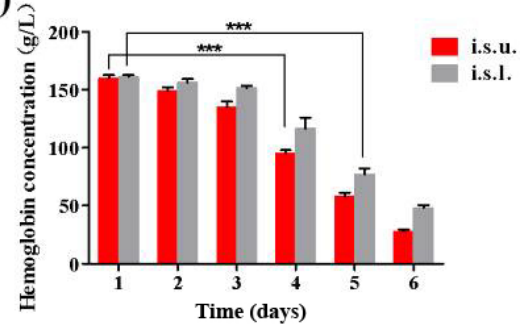

(d)

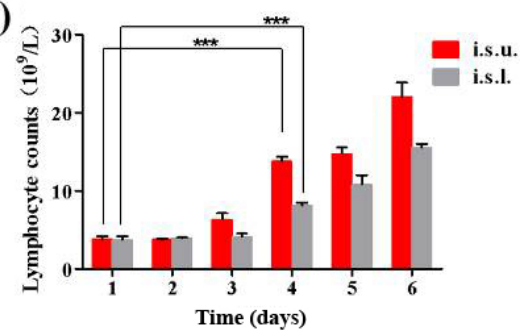

Figure 3 Hematological alterations of interstitial infection. (a) RBC counts. (b) HGB concentration. (c) WBC counts. (d) LYM counts. ${ }^{* * *} p<0.01$. 
on day 4 after infection, and eight times on day 6 .

\subsection{Histopathological alterations of interstitial infection}

The liver is the incubation site for malaria parasites while the spleen plays a key role in eliminating the invasive plasmodia. To better understand the infection process by ISI, liver and spleen sections were stained with hematoxylin and eosin (Fig. 4). Brown hemozoin granules make malaria parasites easily distinguished from surrounding cells. Similar to results from blood smears and hematological parameters, stained sections demonstrated no significant differences between the i.s.l. group and the i.s.u. group on day 3. Large amounts of brown granules could be seen in the liver sections from day 4 post-infection. As expected, the i.s.u. group hid more merozoites in hepatocytes than the i.s.l. group (Fig. 4). These recondite merozoites could be released into blood circulation, resulting in higher parasitemia and higher recurrence rate. On day 6 post-infection, the incubated parasites in the i.s.u. group were three times as much as that of the i.s.l. group. However, an obvious difference in the number of sequestrated parasites in the spleen was only observed on day 3 post-infection. Due to a higher infection rate, more parasites of the i.s.l. group were trapped by the splenic red pulp. As the infection worsened, the spleen of both groups sequestrated more parasites, showing no obvious differences.

Moreover, we compared the ISI group with the i.p. group to assess histopathological alterations caused by differences in injection methods. Interstitial space injection constrained the movement of parasites, causing a gradual invasion of blood vessels, resulting in lower evasion probability and easier recognition and sequestration by the spleen. Sequestrated parasites, although in small amount, can be clearly observed in spleen red pulp of the ISI group at $12 \mathrm{~h}$ post-infection, which was different in the i.p. group. Parasites injected by i.p. could directly invade the liver and incubate, hardly seen in the spleen on the first two days after infection; thus, they were observed in liver sections and not in spleen sections at $12 \mathrm{~h}$ post-infection. As expected, the i.p. group exhibited a larger number of resident merozoites in the liver on day 3 post- infection than the ISI group. This result demonstrates that ISI simulated the natural infection process to some extent, displaying slower histopathological alterations in accordance with natural events.

\subsection{Characterization of ALL}

Artemisinin, a sesquiterpene lactone with an endoperoxide bridge, is known for its antimalarial efficacy [23]. However, its poor aqueous solubility and short blood half-life obstruct its therapeutic efficacy, making drug-loaded carrier a potential way to enhance its bioavailability. We prepared ALL by thin film hydration method and evaluated whether the interstitial space can be utilized for malaria treatment. We characterized the ALL by negative staining using uranyl acetate and visualizing on a TEM (Fig. 5(a)). TEM image showed that the liposomes were spherical in shape with good monodispersity. The mean hydrodynamic diameter measured by dynamic light scattering was $107.3 \mathrm{~nm}$ with a polydispersity of $0.19 \pm 0.08$ (Fig. 5(b)).

\subsection{Decreased parasitemia after interstitial adminis- tration}

Interstitial space had been reported as a pathway for the transportation of fluid into and out of the brain, which was related with the pathophysiology of neurodegenerative diseases [24-26]. Nanoparticles injected in interstitial space can reach organs faster than that injected by i.v., demonstrating a fast and direct transport pathway associated with organs [6]. Herein, we compared therapeutic efficacy in malaria parasite-infected mice treated with ALL by interstitial space injection and oral administration (p.o.) of artemisinin.

High parasitemia $(30 \%-35 \%)$ was modeled by i.p. to demonstrate the significant difference between the ISI group and the p.o. group. Blood smears were performed daily to monitor the alterations of infection rate after drug treatment (Fig. 5(c)). On the first day after administration, all groups exhibited a reduction of malaria parasites, but obvious differences among the two groups were observed. The p.o. group showed

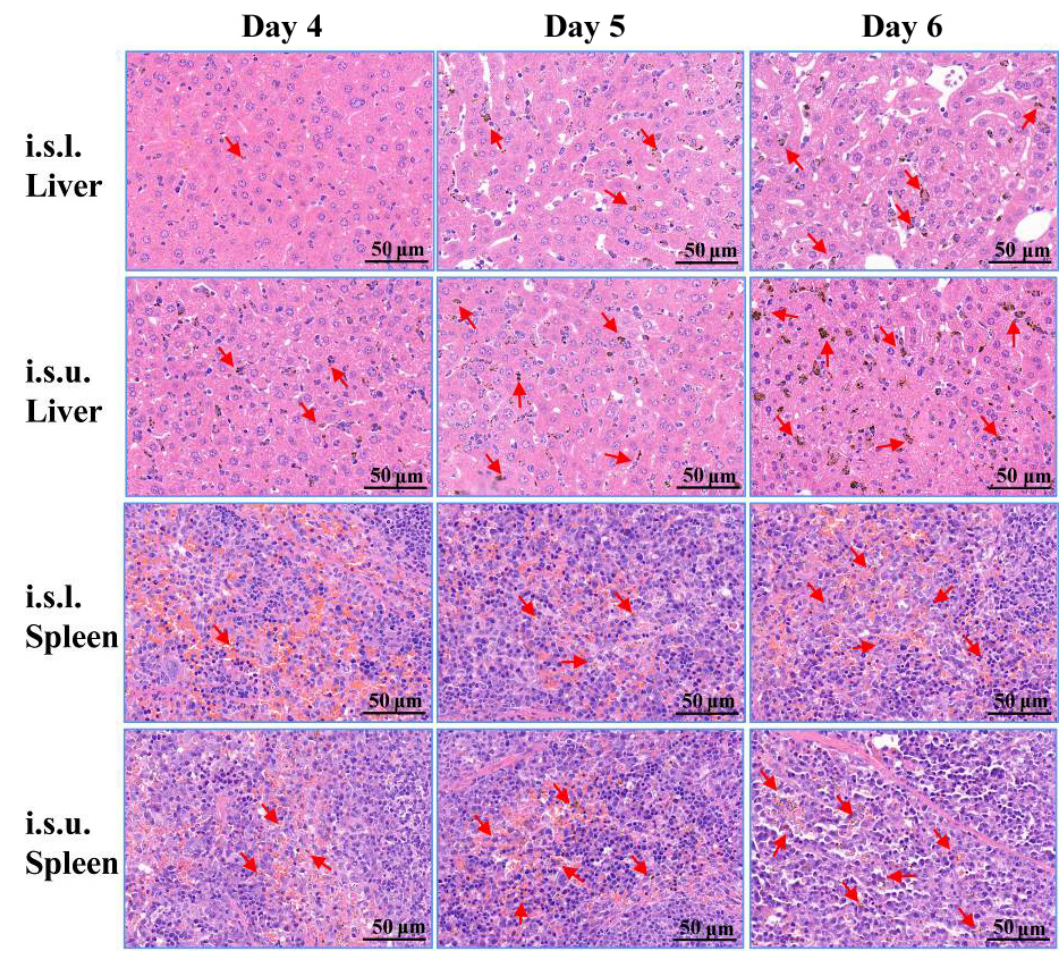

Figure 4 Histopathological alterations of interstitial injection. Liver and spleen sections stained with hematoxylin and eosin at 4-6 days post-infection. Sequestrated malarial parasites are marked with red arrows. 


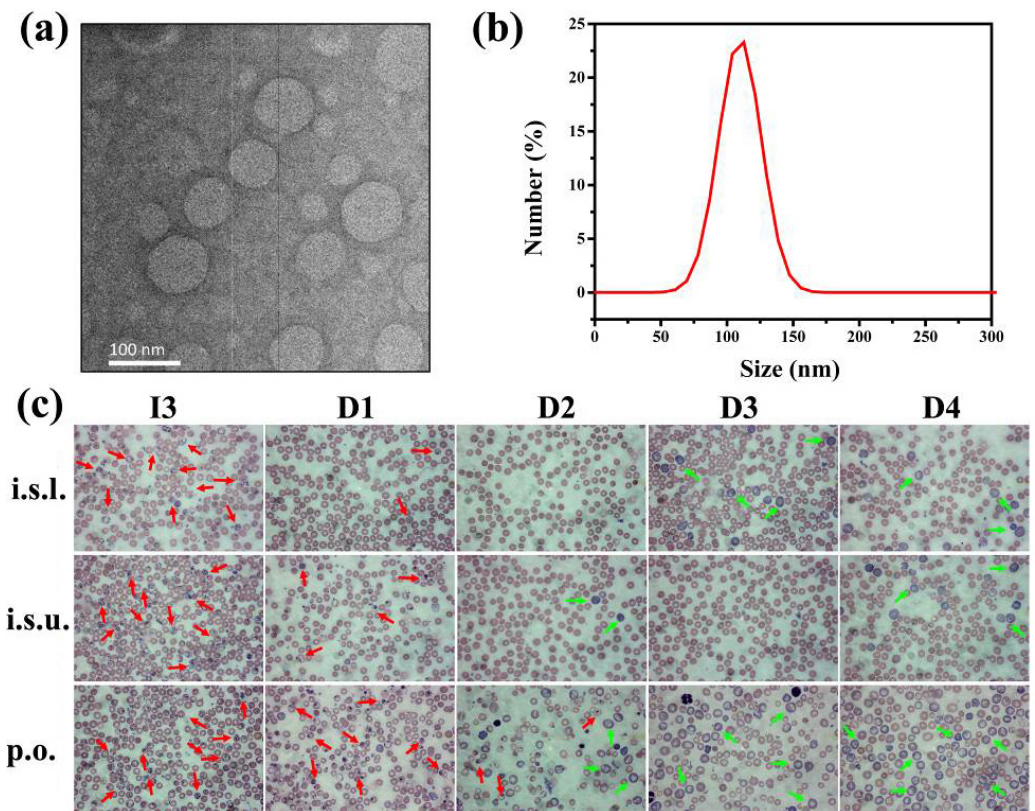

Figure 5 Interstitial administration of ALL for malaria treatment. (a) TEM images of ALL. (b) Hydrodynamic diameter of ALL measured by dynamic light scattering. (c) Blood smear images after treatment by different routes of administration. Infected red blood cells are marked with red arrow. I3: day 3 after infection. D1-D4: days after treatment.

a slightly decreased infection rate with visible mature schizonts, which indicated the incomplete clearance of parasites within the pharmacodynamic time of artemisinin. Unexpectedly, the ISI group showed a better therapeutic effect with a largely decreased infection rate and insignificant number of matured parasites. This could be attributed to the longer circulation time of the liposome and its controlled release by the perivascular space. In the i.s.l. group, only individual merozoites dissociated outside RBCs, possibly attempting to invade the RBCs or freshly entered into cells, having been newly released from a hidden sanctuary. As for the i.s.u. group, a few schizonts stage can be seen in blood smear images and the amounts of infected erythrocytes were slightly more than the i.s.l. group. On day 2 after administration, the i.s. group showed clean images with normal biconcave RBCs and invisible merozoites while the p.o. group still had visible merozoites inside erythrocytes.

Due to high first pass metabolism and short half-life (1-1.5 h), artemisinin could only kill parasites in blood circulation but not the hidden ones, resulting in high infection recurrence rate, and blocking of blood vessels and perivascular spaces upon release of the hidden parasites from the liver. From day 3 after treatment, iRBCs were barely visible in all three groups. However, a few free parasites were observed outside erythrocytes, which indicated that the hidden parasites were gradually being released into blood circulation, although the blood smears presented no iRBCs a day before. Polychromatic red cells (grayish blue), or immature red blood cells, appeared in blood smear after treatment, indicating the strong hematopoietic function of bone marrow and the high activity of RBCs production. Herein, we demonstrated that the treatment by interstitial space exhibited a better therapeutic effect than the oral administration, which should be taken into consideration as a potential therapy route.

\subsection{Improved hematological parameters after interstitial administration}

We further assessed hematological parameters to monitor the recovery status of infected mice after artemisinin treatment (Fig. 6). Main indicators of blood test, including RBC count,
HGB concentration, WBC count, and LYM counts, were statistically compared in different treatment delivery methods. In the first three days, the p.o. group exhibited high infection rate, and large amounts of iRBCs were destroyed and eliminated after drug administration. Consequently, rather than increasing, the RBCs number of the p.o. group decreased in the first three days post-administration. Four days post-administration, an RBCs counts tend to increase in the p.o. group, which was consistent with blood smear. In contrast, the ISI group exhibited a steady increase in RBC count and HGB concentration, which returned to normal levels after a one-week treatment regime. Further, the ISI group showed higher WBC and LYM counts than the p.o. group on days 3 and 4 after treatment, which indicated a better lethal effect on parasites. Based on these results, we concluded that artemisinin treatment by ISI exhibited a better blood restorative capacity and a better regulation effect on immunocytes in malaria parasite-infected mice.

\subsection{Histopathological recovery after interstitial administration}

After the infected mice were treated with ALL by interstitial space injection, the liver and spleen were sectioned and stained by hematoxylin and eosin to evaluate the recovery status of these organs (Fig. 7(a)). As the liver is the incubation site for parasites, the more the number of parasites hidden in the liver, the greater the chance of recurrence. Compared with liver sections from infected mice without drug treatment, drug treatment resulted in largely decreased number of parasites resident in liver. With time, fewer parasites resided in liver. There were still some visible parasites present in liver on day 5 post-administration, coinciding with the appearance of blue RBCs on blood smear images. After one week of drug administration, parasites were hardly seen in liver. Similar to the liver, spleen also started returning to normal. Observations on the first day of drug administration revealed large amounts of sequestrated parasites in spleen red pulp, reflecting the decline in blood circulation. After several days of treatment, the number of parasites trapped in the spleen decreased largely, consistent with the diminishing 


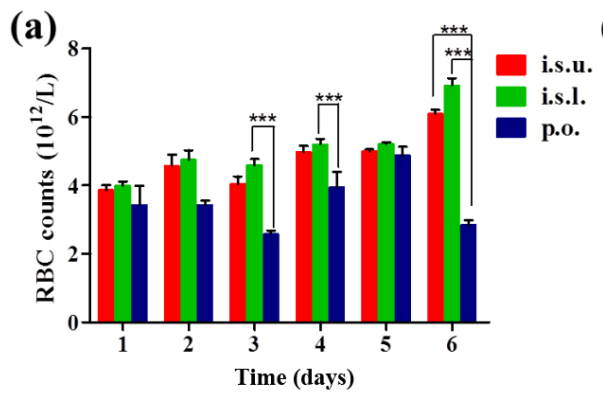

(b)
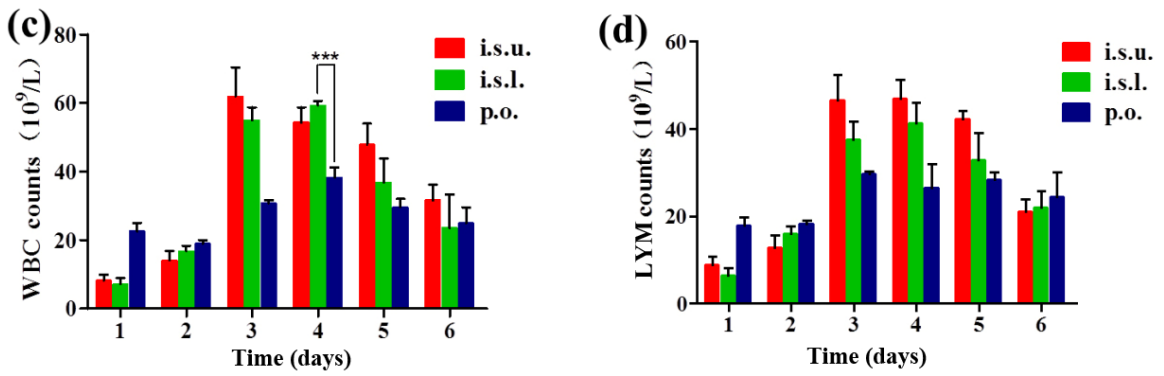

Figure 6 Improved hematological parameters by interstitial administration. (a) RBC count. (b) HGB concentration. (c) WBC count. (d) LYM count. $* * * p<0.01$.

infection rate.

Moreover, we compared sections from the ISI group with the p.o. group. As already known, malaria parasites can escape from being killed by immunocytes expressing proteins. Hence, plasmodia can live in hepatocytes free of immune attack (Fig. 7(b)). After artemisinin treatment by p.o., a few immunocytes could be seen around the parasites in the liver. In contrast, we observed a large number of immunocytes surrounding plasmodia in the liver sections from ISI group, indicating that interstitial space treatment activated and recruited immunocytes to facilitate the elimination of parasites resident in the liver (Fig. 7(c)).

\subsection{Increased plasma concentration after interstitial administration}

Next, we analyzed drug plasma concentration after interstitial administration compared with oral administration using LC-MS (Fig. 7(d)). Artemisinin exhibited low water solubility, high first pass effect and short half-life, so that it could not fully exert its efficacy after oral administration. Plasma levels of artemisinin reached peak value around $0.5 \mathrm{~h}$, and decreased gradually in p.o.. The plasma concentration of artemisinin after oral administration was negligible after $4 \mathrm{~h}$ post-dosing.

In interstitial administration group, we treated mice with (a)

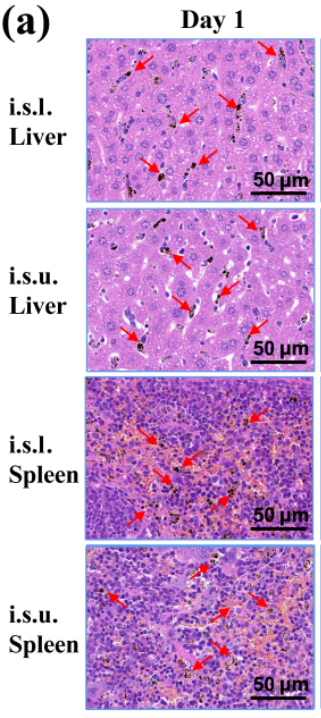

(b)

Liver

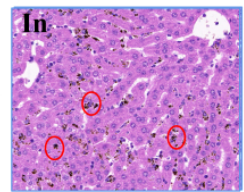

Day 3

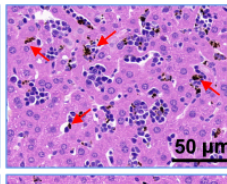

Day 5

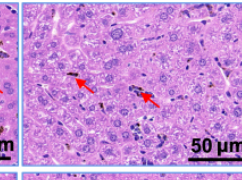

(c)

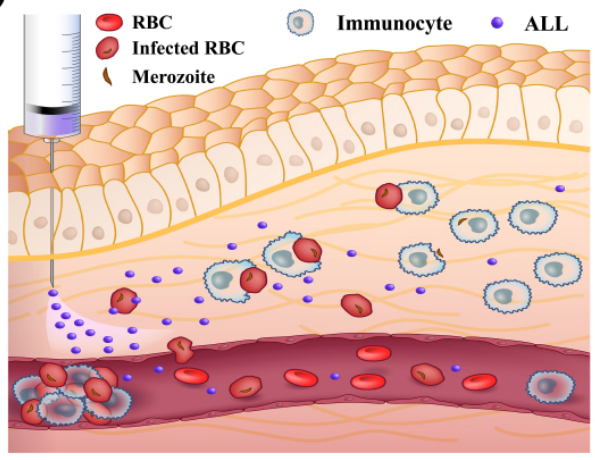

(d)

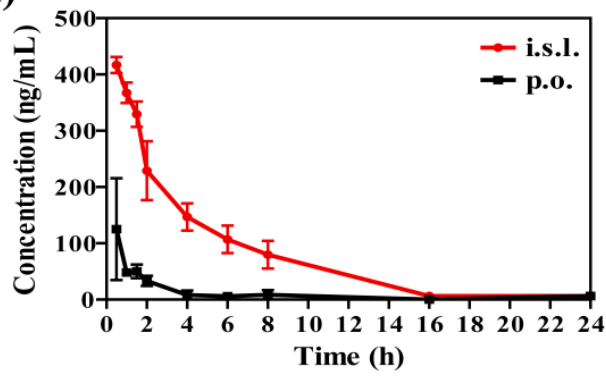

Figure 7 Histopathological recovery after interstitial treatment. (a) Liver and spleen sections stained with hematoxylin and eosin at days 1, 3, and 5 after drug administration. Sequestrated malarial parasites are marked with red arrows. (b) Liver section before drug treatment without immunocytes surrounding parasites (brown, red circles) (left); liver section on day 3 after treatment by p.o., with few immunocytes surrounding the parasites (brown, red circles) (middle); liver section on day 3 after treatment by ISI with a large number of immunocytes surrounding the parasites (brown, red circles). (c) Schematic diagram of interstitial administration. (d) Plasma concentration-time profile of artemisinin in mice. 
equivalent ALL. Significant difference could be seen between p.o. and i.s.l. groups. At $0.5 \mathrm{~h}$ post-dosing, the plasma concentration of interstitial group reached the highest value, three folds higher than oral administration. Notably, interstitial administration resulted in higher plasma concentration than oral administration over a long dosing period, and artemisinin was detectable even after $8 \mathrm{~h}$ of administration, which contributes to the rapid decrease in the number of malaria parasites.

\section{Discussions}

Current mechanism of recurrence has been ascribed to incomplete elimination of parasites in the liver and blood, ignoring the importance of the presence of interstitial space. Based on our findings in the current study, we propose an additional recurrence model, in which parasites sequestrated in interstitial space can traverse through endothelial cells into the vascular lumen and start their new life cycle. As mentioned, parasitized erythrocytes can slip into interstitial space due to disrupted vascular integrity and vascular hemorrhage, providing a refuge for parasites from immune detection and drug killing. Considering its short half-life and suboptimal concentration, it is difficult for artemisinin to reach the interstitial space. The parasites inside erythrocytes can continue to grow and mature until the cells break and release merozoites into the interstitial space. These merozoites can invade normal erythrocytes in interstitial space or traverse through the endothelial cells into blood circulation. Besides, flattened interstitial connection makes it easier for merozoites to arrive at the capillary network. As known, capillaries are the main sites of substance exchange between blood and surrounding tissues. Capillary permeability plays vital role in controlling the substance exchange between blood plasma and interstitial fluid [27]. The permeability depends on the structure of capillaries, such as endothelial intercellular junctions, plasmalemmal vesicles and fenestrae [28]. These open intercellular junctions and fenestrae permit direct contact between blood and interstitial fluid, resulting in the fusion of capillary network and interstitial stream. Thus, it is easier for merozoites to invade blood vessels at the terminal vascular bed, not only because of the thin capillary wall, but also because of this fusion.

In addition to being a sanctuary for mature merozoites, interstitial space also played a vital role at the beginning of infection. The infection process is initially triggered when Plasmodium sporozoites are injected into the skin of the mammalian host by the bite of Anopheles mosquito. The sporozoites travel from the bite site to the liver, where they invade hepatocytes and differentiate into merozoites $[29,30]$. The journey of sporozoites includes penetrating cell barriers, migrating into dermal space, entering blood vessels, and crossing liver sinusoids [31]. The perivascular arena provides two possibilities of gliding motility to parasites-avascular motility and perivascular motility [32]. Outside the perivascular space, the initial avascular motility of parasites is random over a large area, increasing the probability of parasites encountering blood vessels. However, the movement inside the perivascular space is more constrained and tends to follow the curvature of blood vessels, which makes it easier for parasites to enter the vessels [32]. Only a proportion of sporozoites successfully exits the dermis and enters blood vessels, whereas $20 \%$ are drained by lymphatics [33,34]. Moreover, some sporozoites are engulfed by phagocytic cells in the dermis, while the rest can avoid being killed by traversing the epithelial cell and remaining in the dermal interstitium, maturing into intracellular stages [35,
36]. Parasites that survive by residing in interstitial space later invade blood vessels and complete their erythrocytic stage. Therefore, in-depth study of the role of interstitial space at the beginning and after infection of malaria is of great significance for malaria prevention and treatment.

During malaria treatment, we found that interstitial administration could not only prolonged drug blood circulation time but also increased the drug plasma concentration, which greatly improve the efficacy of artemisinin. In addition to enhancing drug efficacy compared to oral administration, drug given by interstitial space may also decrease the nanotoxicity caused by direct injection of nanoparticles into the blood. The selective permeability of vascular walls and the reticuloendothelial system makes disease therapy by interstitial administration safer. At the same time, interstitial administration may recruit immunocytes to facilitate the elimination of retained parasites. It has been reported that macrophages can enter into the interstitial space in response to the presence of foreign materials [3]. T cells can be recruited to the perivascular space during the development of experimental cerebral malaria [18]. Hence, interstitial treatment could be applied to activate immune response and invalidate immune evasion of malaria parasites. The mechanism of immunocytes recruitment during interstitial treatment is still unclear, which needs further studies. The injection of allogenic nanoparticles may activate autoimmunity, which may account for the immunocytes recruitment. Moreover, the signal transmission of nervous system may be affected by interstitial administration, which further modulates immune response. Similar with the fusion of capillary and interstitial stream, the transmission of neurotransmitters between neural network and tissue cells is accomplished in interstitial space. The communication between muscle and nerve depends on the neuromuscular junction, a synaptic connection where nerve ending contacts muscle fibers [37]. The neuromuscular junction is compose of nerve terminal, muscle fiber, and the non-myelinating Schwann cells, transmitting acetylcholine from neuron terminals to receptors on muscle fibers in interstitial space [38]. Drugs by interstitial administration may directly influence signal transmission or give another feedback to nervous system, resulting in immunoregulation in response to nerve activation.

Similar to Plasmodium, other infectious agents, such as bacteria, virus, other parasites, may also be closely related to the interstitial space. The invasion of bacteria through an epithelial barrier activated interstitium resident macrophages, leading to recruitment of leukocytes into interstitial space and systemic immune responses [39]. Bacteria can evade host defense by secreting hemolysin to deplete perivascular macrophages, effectively preventing the recruitment of neutrophils into interstitium [40]. Virus may also disseminate in interstitial spaces surrounding blood vessels [41]. Hence, blocking immune escape and reducing retention in the interstitial space is vital for treating infectious diseases.

In addition to dissemination in blood and interstitial stream, perineural spread is another pathway for tumor and neurotropic pathogens invasion in human. Tumors of head and neck can spread along the trigeminal and facial nerves, which can be clinically undetectable [42]. The diagnosis and treatment for perineural tumor spread are lacking and controversial due to unsuspected manifestation. Besides, a transcribrial route has been described, by which parasites and virus can reach brain from olfactory bulb along olfactory modulating nerves $[43,44]$. The coronavirus infectious disease 2019 (COVID-19) may also exploit this transnasal pathway to 
affect the brain [45]. From this aspect, interstitial administration may be a potential way to treat perineural spread of tumors and virus.

\section{Conclusions}

In conclusion, we verified the infectivity of parasites sequestrated in interstitial space during interstitial infection, complementing infection recurrence. Compared with intravenous and intraperitoneal infection, interstitial infection exhibited a different infection rate and pathological alteration by including the perivascular space into the infection process. Further, we found that interstitial treatment unexpectedly activated and recruited a large amount of immunocytes to surround parasites resident in the liver, facilitating the elimination of parasites in malaria parasite-infected mice treated with ALL. Compared with oral administration, interstitial administration of artemisinin resulted in largely decreased infection rate and enhanced pathological recovery. This interstitial route lengthened the blood circulation time of artemisinin and increased its plasma levels, making up for the inefficiency of oral administration and the nanotoxicity of intravenous administration, thus providing a potential strategy for infectious disease therapy.

\section{Acknowledgements}

This work was supported by the National Natural Science Foundation of China (Nos. 81641002, 81841001 and 31971245), and Key Research Program of Frontier Sciences of CAS (No. ZDBS-LY-SLH036).

\section{References}

[1] Hu, N.; Shi, X. L.; Zhang, Q.; Liu, W. T.; Zhu, Y. T.; Wang, Y. Q.; Hou, Y.; Ji, Y. L.; Cao, Y. P.; Zeng, Q. et al. Special interstitial route can transport nanoparticles to the brain bypassing the blood-brain barrier. Nano Res. 2019, 12, 2760-2765.

[2] Venkatesh, B.; Morgan, T. J.; Cohen, J. Interstitium: The next diagnostic and therapeutic platform in critical illness. Crit. Care Med. 2010, 38, S630-S636.

[3] Wiig, H.; Rubin, K.; Reed, R. K. New and active role of the interstitium in control of interstitial fluid pressure: Potential therapeutic consequences. Acta Anaesthesiol. Scand. 2003, 47, 111-121.

[4] Benias, P. C.; Wells, R. G.; Sackey-Aboagye, B.; Klavan, H.; Reidy, J.; Buonocore, D.; Miranda, M.; Kornacki, S.; Wayne, M.; Carr-Locke, D. L. et al. Structure and distribution of an unrecognized interstitium in human tissues. Sci. Rep. 2018, 8, 4947.

[5] Kumar, A.; Ghosh, S. K.; Faiq, M. A.; Deshmukh, V. R.; Kumari, C.; Pareek, V. A brief review of recent discoveries in human anatomy. QJM: An Int. J. Med. 2019, 112, 567-573.

[6] Hu, N.; Cao, Y. P.; Ao, Z.; Han, X. X.; Zhang, Q.; Liu, W. T.; Liu, S. D.; Liao, F. L.; Han, D. Flow behavior of liquid metal in the connected fascial space: Intervaginal space injection in the rat wrist and mice with tumor. Nano Res. 2018, 11, 2265-2276.

[7] Shi, X. L.; Zhu, Y. T.; Hua, W. D.; Ji, Y. L.; Ha, Q.; Han, X. X.; Liu, Y.; Gao, J. W.; Zhang, Q.; Liu, S. D. et al. An in vivo study of the biodistribution of gold nanoparticles after intervaginal space injection in the tarsal tunnel. Nano Res. 2016, 9, 2097-2109.

[8] Sicard, R. E.; Nguyen, L. M. P. Interstitial fluids associated with wound repair support proliferation but not differentiation of neonatal rat myoblasts in vitro. Wound Repair Regen. 1994, 2, 306-313.

[9] Correa-Gallegos, D.; Jiang, D. S.; Christ, S.; Ramesh, P.; Ye, H. F.; Wannemacher, J.; Gopal, S. K.; Yu, Q.; Aichler, M.; Walch, A. et al. Patch repair of deep wounds by mobilized fascia. Nature 2019, 576, 287-292.

[10] Wardlaw, J. M.; Benveniste, H.; Nedergaard, M.; Zlokovic, B. V.; Mestre, H.; Lee, H.; Doubal, F. N.; Brown, R.; Ramirez, J.; MacIntosh, B. J. et al. Perivascular spaces in the brain: Anatomy, physiology and pathology. Nat. Rev. Neurol. 2020, 16, 137-153.

[11] Rasmussen, M. K.; Mestre, H.; Nedergaard, M. The glymphatic pathway in neurological disorders. Lancet Neurol. 2018, 17, 1016-1024.

[12] Kim, Y. K.; Nam, K. I.; Song, J. The glymphatic system in diabetesinduced dementia. Front. Neurol. 2018, 9, 867.

[13] Jiang, Q.; Zhang, L.; Ding, G. L.; Davoodi-Bojd, E.; Li, Q. J.; Li, L.; Sadry, N.; Nedergaard, M.; Chopp, M.; Zhang, Z. G. Impairment of the glymphatic system after diabetes. J. Cereb. Blood Flow Metab. 2017, 37, 1326-1337.

[14] Tarasoff-Conway, J. M.; Carare, R. O.; Osorio, R. S.; Glodzik, L.; Butler, T.; Fieremans, E.; Axel, L.; Rusinek, H.; Nicholson, C.; Zlokovic, B. V. et al. Clearance systems in the brain-implications for Alzheimer disease. Nat. Rev. Neurol. 2015, 11, 457-470.

[15] Promeneur, D.; Lunde, L. K.; Amiry-Moghaddam, M.; Agre, P. Protective role of brain water channel AQP4 in murine cerebral malaria. Proc. Natl. Acad. Sci. USA 2013, 110, 1035-1040.

[16] Fedosov, D. A.; Caswell, B.; Suresh, S.; Karniadakis, G. E. Quantifying the biophysical characteristics of Plasmodium-falciparum-parasitized red blood cells in microcirculation. Proc. Natl. Acad. Sci. USA 2011, 108, 35-39.

[17] Coban, C.; Lee, M. S. J.; Ishii, K. J. Tissue-specific immunopathology during malaria infection. Nat. Rev. Immunol. 2018, 18, 266-278.

[18] Shaw, T. N.; Stewart-Hutchinson, P. J.; Strangward, P.; Dandamudi, D. B.; Coles, J. A.; Villegas-Mendez, A.; Gallego-Delgado, J.; van Rooijen, N.; Zindy, E.; Rodriguez, A.; Brewer, J. M. et al. Perivascular arrest of $\mathrm{CD} 8^{+} \mathrm{T}$ cells is a signature of experimental cerebral malaria. PLoS Pathog. 2015, 11, e1005210.

[19] Lackner, P.; Beer, R.; Helbok, R.; Broessner, G.; Engelhardt, K.; Brenneis, C.; Schmutzhard, E.; Pfaller, K. Scanning electron microscopy of the neuropathology of murine cerebral malaria. Malar. J. 2006, 5, 116.

[20] De Niz, M.; Nacer, A.; Frischknecht, F. Intravital microscopy: Imaging host-parasite interactions in the brain. Cell. Microbiol. 2019, 21, e13024.

[21] Nacer, A.; Movila, A.; Baer, K.; Mikolajczak, S. A.; Kappe, S. H. I.; Frevert, U. Neuroimmunological blood brain barrier opening in experimental cerebral malaria. PLoS Pathog. 2012, 8, e1002982.

[22] Karch, R.; Neumann, F.; Podesser, B. K.; Neumann, M.; Szawlowski, P.; Schreiner, W. Fractal properties of perfusion heterogeneity in optimized arterial trees: A model study. J. Gen. Physiol. 2003, 122, 307-321.

[23] Klayman, D. L. Qinghaosu (artemisinin): An antimalarial drug from China. Science 1985, 228, 1049-1055.

[24] Thrane, V. R.; Thrane, A. S.; Plog, B. A.; Thiyagarajan, M.; Iliff, J. J.; Deane, R.; Nagelhus, E. A.; Nedergaard, M. Paravascular microcirculation facilitates rapid lipid transport and astrocyte signaling in the brain. Sci. Rep. 2013, 3, 2582.

[25] Iliff, J. J.; Wang, M. H.; Liao, Y. H.; Plogg, B. A.; Peng, W. G.; Gundersen, G. A.; Benveniste, H.; Vates, G. E.; Deane, R.; Goldman, S. A. et al. A paravascular pathway facilitates CSF flow through the brain parenchyma and the clearance of interstitial solutes, including Amyloidß. Sci. Transl. Med. 2012, 4, 147ra111.

[26] Ahn, J. H.; Cho, H.; Kim, J. H.; Kim, S. H.; Ham, J. S.; Park, I.; Suh, S. H.; Hong, S. P.; Song, J. H.; Hong, Y. K. et al. Meningeal lymphatic vessels at the skull base drain cerebrospinal fluid. Nature 2019, 572, 62-66.

[27] Stan, R. V.; Tse, D.; Deharvengt, S. J.; Smits, N. C.; Xu, Y.; Luciano, M. R.; McGarry, C. L.; Buitendijk, M.; Nemani, K. V.; Elgueta, R. et al. The diaphragms of fenestrated endothelia: Gatekeepers of vascular permeability and blood composition. Dev. Cell 2012, 23, $1203-1218$.

[28] Casley-Smith, J. R. Functioning and interrelationships of blood capillaries and lymphatics. Experientia 1976, 32, 1-12.

[29] Cowman, A. F.; Healer, J.; Marapana, D.; Marsh, K. Malaria: Biology and disease. Cell 2016, 167, 610-624.

[30] Cowman, A. F.; Crabb, B. S. Invasion of red blood cells by malaria parasites. Cell 2006, 124, 755-766.

[31] Yamauchi, L. M.; Coppi, A.; Snounou, G.; Sinnis, P. Plasmodium sporozoites trickle out of the injection site. Cell. Microbiol. 2007, 9, $1215-1222$. 
[32] Hopp, C. S.; Chiou, K.; Ragheb, D. R. T.; Salman, A. M.; Khan, S. M.; Liu, A. J.; Sinnis, P. Longitudinal analysis of Plasmodium sporozoite motility in the dermis reveals component of blood vessel recognition. eLife 2015, 4, e07789.

[33] Amino, R.; Giovannini, D.; Thiberge, S.; Gueirard, P.; Boisson, B.; Dubremetz, J. F.; Prévost, M. C.; Ishino, T.; Yuda, M.; Ménard, R. Host cell traversal is important for progression of the malaria parasite through the dermis to the liver. Cell Host Microbe 2008, 3, $88-96$.

[34] Sinnis, P.; Zavala, F. The skin stage of malaria infection: Biology and relevance to the malaria vaccine effort. Future Microbiol. 2008, 3, 275-278.

[35] Amino, R.; Thiberge, S.; Martin, B.; Celli, S.; Shorte, S.; Frischknecht, F.; Ménard, R. Quantitative imaging of Plasmodium transmission from mosquito to mammal. Nat. Med. 2006, 12, 220-224.

[36] Ejigiri, I.; Sinnis, P. Plasmodium sporozoite-host interactions from the dermis to the hepatocyte. Curr. Opin. Microbiol. 2009, 12, 401-407.

[37] Lepore, E.; Casola, I.; Dobrowolny, G.; Musarò, A. Neuromuscular junction as an entity of nerve-muscle communication. Cells 2019, $8,906$.

[38] Alvarez-Suarez, P.; Gawor, M.; Prószyński, T. J. Perisynaptic schwann cells-The multitasking cells at the developing neuromuscular junctions. Semin. Cell Dev. Biol. in press, DOI:10.1016/j.semcdb.2020.02.011.
[39] Munford, R. S. Severe sepsis and septic shock: The role of gram-negative bacteremia. Annu. Rev. Pathol.: Mech. Dis. 2006, 1, 467-496.

[40] Abtin, A.; Jain, R.; Mitchell, A. J.; Roediger, B.; Brzoska, A. J.; Tikoo, S.; Cheng, Q.; Ng, L. G.; Cavanagh, L. L.; von Andrian, U. H. et al. Perivascular macrophages mediate neutrophil recruitment during bacterial skin infection. Nat. Immunol. 2014, 15, 45-53.

[41] Couderc, T.; Chrétien, F.; Schilte, C.; Disson, O.; Brigitte, M.; Guivel-Benhassine, F.; Touret, Y.; Barau, G.; Cayet, N.; Schuffenecker, I. et al. A mouse model for chikungunya: Young age and inefficient type-I interferon signaling are risk factors for severe disease. PLoS Pathog. 2008, 4, e29.

[42] Veness, M. J.; Biankin, S. Perineural spread leading to orbital invasion from skin cancer. Australas. Radiol. 2000, 44, 296-302.

[43] Baig, A. M. Primary amoebic meningoencephalitis: Neurochemotaxis and neurotropic preferences of Naegleria fowleri. ACS Chem. Neurosci. 2016, 7, 1026-1029.

[44] Netland, J.; Meyerholz, D. K.; Moore, S.; Cassell, M.; Perlman, S. Severe acute respiratory syndrome coronavirus infection causes neuronal death in the absence of encephalitis in mice transgenic for human ACE2. J. Virol. 2008, 82, 7264-7275.

[45] Baig, A. M.; Khaleeq, A.; Ali, U.; Syeda, H. Evidence of the COVID-19 virus targeting the CNS: Tissue distribution, host-virus interaction, and proposed neurotropic mechanisms. ACS Chem. Neurosci. 2020, 11, 995-998. 